\title{
A nearly optimal Galerkin projected residual finite element method for Helmholtz problem
}

\author{
Eduardo Gomes Dutra do Carmo ${ }^{\mathrm{a}, *}$, Gustavo Benitez Alvarez ${ }^{\mathrm{b}}$, \\ Abimael Fernando Dourado Loula ${ }^{c}$, Fernando Alves Rochinha ${ }^{a}$ \\ ${ }^{a}$ COPPE - Universidade Federal do Rio de Janeiro, Ilha do Fundão, P.B. 68509, Rio de Janeiro 21945-970, RJ, Brazil \\ ${ }^{\mathrm{b}}$ UFF/EEIMVR - Universidade Federal Fluminense, Av. dos Trabalhadores 420, Volta Redonda 27225-125, RJ, Brazil \\ ${ }^{\mathrm{c}}$ LNCC - Laboratorio Nacional de Computac̃ão Científica, Av. Getúlio Vargas 333, P.B. 95113, Petrópolis 25651-070, RJ, Brazil
}

Received 30 June 2007; received in revised form 1 November 2007; accepted 12 November 2007

Available online 22 November 2007

\begin{abstract}
A Finite Element Formulation for scalar and linear second-order boundary value problems is introduced. The new method relies on a variational formulation obtained following the usual path of appending to the Galerkin variational formulation, a balanced residual form of the governing partial differential equation computed within each element. The novelty consists of projecting the residual in a subspace defined for each element, which gives rise to the name of the method: Galerkin Projected Residual (GPR). This subspace is built by systematically exploring some a priori criteria (either based on the physics or on the underlying mathematics). The method can be used to stabilize a variety of problems. Here it is applied to Helmholtz equation, where standard Galerkin formulations are known to present poor approximations for high wave numbers. The method is formally introduced along with some numerical examples that are used to assess the improvements achieved.
\end{abstract}

(C) 2007 Elsevier B.V. All rights reserved.

Keywords: Finite element method; Stabilization; GPR; GLS; GGLS; Helmholtz equation

\section{Introduction}

Numerical approximation of time-harmonic acoustic, elastic and electromagnetic wave problems governed by the Helmholtz equation is particularly challenging as reported in a vast literature [1-20]. The oscillatory behavior of the exact solution and the quality of the numerical approximation depend on the wave number $k$. To approximate Helmholtz equation with acceptable accuracy the resolution of the mesh should be adjusted to the wave number according to a rule of thumb [1], which prescribes a minimum number of elements per wavelength. Despite this rule, the performance of the Galerkin finite element

\footnotetext{
* Corresponding author.

E-mail addresses: carmo@con.ufrj.br (E.G. Dutra do Carmo), benitez.gustavo@gmail.com (G.B. Alvarez), aloc@Incc.br (A.F.D. Loula), rochinha@adc.coppe.ufrj.br (F.A. Rochinha).
}

method deteriorates as $k$ increases. This misbehavior, known as pollution of the finite element solution, can only be avoided after a drastic refinement of the mesh, which normally entails significant barriers for the numerical analysis of Helmholtz equation at mid and high frequencies.

A great effort has been devoted to alleviate the pollution effect. There exist several attempts to minimize the phase error of finite element approximations to Helmholtz equation. In one-dimension, a Galerkin Least Square (GLS) stabilization, as proposed in [10], can completely eliminate the phase error, but not in two or three-dimensions $[5,6]$. For two-dimensions, stencils with minimal pollution error are constructed in [3] through the Quasi Stabilized Finite Element Method (QS). As the Quasi-Stabilized Finite Element Method is not based on a variational formulation it is not clear how this formulation can be applied to non-uniform meshes, high-order polynomials and non-homogeneous problems. Finite element methods based on variational 
formulations, such as Residual-Based Finite Element Method (RBFEM) [7] and Discontinuous Finite Element Method at Element Level (DGB) [8,9], have also been developed to minimize the phase error in two-dimensions.

The RBFEM method is obtained from the Galerkin approximation by appending terms that are proportional to residuals in element interiors and on inter-element boundaries. The terms proportional to the residuals on the elements can be understood as an extension of GLS method with stabilization parameters dependent on the position. The residuals on inter-element boundaries are motivated by the variational multi-scale formulation. These terms implicate in an extra computational effort compared with a classical continuous finite element formulation. However, this extra computational effort in computing residuals over element boundaries is local to each element and is small relative to the cost of solving global equation systems.

The DGB method is a discontinuous finite element formulation, where discontinuities are introduced locally, inside each element. The discontinuous interpolation functions can be viewed as discontinuous bubbles and the corresponding degrees of freedom can be eliminated at element level by static condensation yielding a global matrix topologically equivalent to those of classical finite element approximations. The free parameters, related to the weak enforcement of continuity inside each element are determined explicitly minimizing the pollution effect. For uniform meshes, the DGB stencil with minimal pollution error is identical to QSFEM stencil derived in [3]. As it is based on a variationally consistent formulation DGB can be naturally applied to non-uniform meshes. In this case, however, it may lead to nonlinear problems at element level associated with the determination of the free parameters and the condensation technique. The local discontinuities introduced on inter-bubbles boundaries also implicate in an extra computational effort.

Our first ideas about a finite element method with multiple projections of residual were developed to address the Helmholtz equation, and are reported in [18]. A generalization of this method (GPR) for scalar and linear secondorder boundary value problems will be presented in [16]. This formulation is obtained adding appropriate projections of the residuals of the differential equation, at element level, to the Galerkin formulation. This formulation is naturally applied to elliptic operators in multidimensions introducing consistently the maximum number of stabilization parameters associated with uniform or non-uniform meshes. Here, this new formulation is applied to Helmholtz equation aiming at minimizing the phase error.

The paper is organized as follows: The model boundaryvalue problem, the associated variational formulation and the Galerkin FEM are briefly presented in Section 2. In Section 3, we present the theoretical framework of the Galerkin Residual Projected finite element method. In Section 4, the GPR method is applied to Helmholtz equation. Explicit values of the free parameters minimizing the phase error are presented in this section via a dispersion analysis. In Section 5, some numerical experiments to evaluate the performance of the new formulation are presented. Finally, Section 6 contains some conclusions and final remarks.

\section{The Helmholtz equation}

Let $\Omega \subset R^{n}(n \geqslant 1)$ be an open bounded domain with a Lipschitz continuous smooth piecewise boundary $\Gamma$. Let $\Gamma_{g}, \Gamma_{q}$ and $\Gamma_{r}$ be subsets of $\Gamma$ satisfying $\Gamma_{g} \cap \Gamma_{q}=$ $\Gamma_{g} \cap \Gamma_{r}=\Gamma_{q} \cap \Gamma_{r}=\emptyset$, and $\Gamma=\Gamma_{g} \cup \Gamma_{q} \cup \Gamma_{r}$. We shall consider the interior Helmholtz problem

$\mathscr{L}(u)=-\nabla \cdot(\nabla u)-k^{2} u=f$ in $\Omega$

with boundary conditions

$u=g$ on $\Gamma_{g}$,

$\nabla u \cdot \hat{\mathbf{n}}=q$ on $\Gamma_{q}$,

$\nabla u \cdot \hat{\mathbf{n}}+\alpha u=r$ on $\Gamma_{r}$,

where $u$ denotes the scalar field that describes timeharmonic acoustic, elastic or electromagnetic steady-state waves. In acoustics, it represents the pressure fluctuations. The coefficient $k \in \mathbb{R}$ is the wave number, $f \in L^{2}(\Omega)$ is the source term, $g \in H^{\frac{1}{2}}\left(\Gamma_{g}\right) \cap C^{0}\left(\Gamma_{g}\right), q \in L^{2}\left(\Gamma_{q}\right)$ and $r \in L^{2}\left(\Gamma_{r}\right)$ are the prescribed boundary conditions. The coefficient $\alpha \in L^{\infty}\left(\Gamma_{r}\right)$ is positive on $\Gamma_{r}$ and $\hat{\mathbf{n}}$ denotes the outward normal unit vector defined almost everywhere on $\Gamma$.

\subsection{The associate variational problem}

Let $S$ and $V$ be defined as

$S=\left\{u \in H^{1}(\Omega) ; u=g\right.$ on $\left.\Gamma_{g}\right\}$

and

$V=\left\{v \in H^{1}(\Omega) ; v=0\right.$ on $\left.\Gamma_{g}\right\}$.

The variational problem associated with the boundary value problem defined by (1) and (2) consists of finding $u \in S$ satisfying the following variational equation:

$A(u, v)=f(v) \quad \forall v \in V$

with

$$
\begin{aligned}
& A(u, v)=\int_{\Omega}\left(\nabla u \cdot \nabla v-k^{2} u v\right) \mathrm{d} \Omega+\int_{\Gamma_{r}} \alpha u v \mathrm{~d} \Gamma, \\
& F(v)=\int_{\Omega} f v \mathrm{~d} \Omega+\int_{\Gamma_{q}} q v \mathrm{~d} \Gamma+\int_{\Gamma_{r}} r v \mathrm{~d} \Gamma \quad \forall v \in V .
\end{aligned}
$$

The main challenge is to find a consistent formulation in continuous or discontinuous finite element spaces, such that, its approximate solution is stable and the closest possible to the exact solution of Helmholtz problem. In this paper we will deal only with $C^{0}$ continuous finite dimensional spaces. 


\subsection{The Galerkin finite element formulation}

Let $M^{h}=\left\{\Omega_{1}, \ldots, \Omega_{n_{e}}\right\}$ be a partition of $\Omega$ into $n_{e}$ nondegenerated finite elements $\Omega_{e}$, such that each $\Omega_{e}$ can be mapped in standard elements by isoparametric mapping satisfying $\Omega_{e} \cap \Omega_{e^{\prime}}=\emptyset$ if $e \neq e^{\prime}$ and $\Omega \cup \Gamma=\cup_{e=1}^{n_{e}}\left(\Omega_{e} \cup\right.$ $\Gamma_{e}$ ), where $\Gamma_{e}$ denotes the boundary of each $\Omega_{e}$.

Let $p \geqslant 1$ be an integer and consider $P^{p}\left(\Omega_{e}\right)$ the space of polynomials of degree less than or equal to $p$ defined over each element. Let

$H^{h}(\Omega)=\left\{\varphi \in H^{1}(\Omega) ; \varphi_{e} \in P^{p}\left(\Omega_{e}\right)\right\}$

and

$H^{\frac{1}{2}, h}\left(\Gamma_{g}\right)=\left\{\phi \in H^{\frac{1}{2}}\left(\Gamma_{g}\right) ;\right.$ such that, $\varphi \in H^{h}(\Omega)$ and $\phi=\varphi$ on $\left.\Gamma_{g}\right\}$

be the finite dimension spaces, and $g^{h}$ the interpolant of $g$ on $H^{\frac{1}{2}, h}\left(\Gamma_{g}\right)$. Defining

$S^{h}=\left\{u \in H^{h}(\Omega) ; u=g^{h}\right.$ on $\left.\Gamma_{g}\right\}$

and

$V^{h}=\left\{v \in H^{h}(\Omega) ; v=0\right.$ on $\left.\Gamma_{g}\right\}$

the Galerkin finite element approximation consists of finding $u^{h} \in S^{h}$ such that

$A\left(u^{h}, v^{h}\right)=F\left(v^{h}\right) \quad \forall v^{h} \in V^{h}$.

For purely diffusive problems, the solution of Galerkin FEM is the best approximation in the energy norm which has pushed the massive use of finite elements in several different applications. On the other hand, it is well know that the standard Galerkin FEM suffer from important drawbacks in some situations, like instability and pollution effects when applied to Helmholtz equation with high wave numbers $[1,3-5]$.

\section{Theoretical framework}

The theoretical framework grounding the GPR formulation is quite generic, it can provide the basis for generating stabilized methods for different problems like, for instance, the Diffusion-Reaction equation [16]. The setting up of the theory emphasizes this generality in the sense that the results are valid for any linear differential operator $\mathscr{L}$. The specialization for the Helmholtz equation, in which the functions $\tau_{e}$ and $\tau_{e}^{g}$, introduced in (5) below, are chosen taking into consideration the characteristics of the problem, is carried out in the following section.

The new method relies on a variational formulation obtained following the usual path of appending to the Galerkin variational formulation (4), a balanced residual form of the partial differential equation computed, inside every element, using the finite element solution $u^{h}$. Formally, it is represented by the following equation:

$$
\begin{aligned}
& A\left(u^{h}, v^{h}\right)+\sum_{e=1}^{n_{e}}\left[\left(\mathscr{L}\left(u_{e}^{h}\right), \tau_{e} \hat{\mathscr{L}}\left(v_{e}^{h}\right)\right)_{\Omega_{e}}\right. \\
& \left.\quad+\int_{\Omega_{e}} \tau_{e}^{g} \nabla \mathscr{L}\left(u_{e}^{h}\right) \cdot \nabla \hat{\mathscr{L}}^{g}\left(v_{e}^{h}\right) \mathrm{d} \Omega\right]=F_{\mathrm{GPR}}\left(v^{h}\right) \quad \forall v^{h} \in V^{h}
\end{aligned}
$$

where $\hat{\mathscr{L}}$ and $\hat{\mathscr{L}}^{g}$ are two linear operators on $v_{e}^{h}, \tau_{e}$ and $\tau_{e}^{g}$ are two functions defined on the elements,

$$
\begin{aligned}
F_{\mathrm{GPR}}\left(v^{h}\right)= & F\left(v^{h}\right)+\sum_{e=1}^{n_{e}}\left[\left(f, \tau_{e} \hat{\mathscr{L}}\left(v_{e}^{h}\right)\right)_{\Omega_{e}}\right. \\
& \left.+\int_{\Omega_{e}} \tau_{e}^{g} \nabla f_{e} \cdot \nabla \hat{\mathscr{L}}^{g}\left(v_{e}^{h}\right) \mathrm{d} \Omega\right]
\end{aligned}
$$

with

$\left(u_{e}^{h}, v_{e}^{h}\right)_{\Omega_{e}}=\int_{\Omega_{e}} u_{e}^{h} v_{e}^{h} \mathrm{~d} \Omega$,

and the residual gradient term in (5) is inspired in GGLS method [21].

The linear functional $F_{\mathrm{GPR}}\left(v_{h}\right)$ is an extension of the right side of the Galerkin formulation which takes into account the source term associate with the residual of the partial differential equation. The operators $\hat{\mathscr{L}}, \hat{\mathscr{L}}^{g}$ and the functions $\tau_{e}, \tau_{e}^{g}$ represent the core of the proposed formulation. The approximate solution is still sought in standard $C^{0}$ Lagrangian finite element spaces, but the resulting discrete problem is clearly different from the one obtained departing from the Galerkin formulation. The main goal is to improve the finite element approximation, particularly for originally unstable Galerkin formulations, without increasing the number of degrees of freedom. As the appended terms are only defined locally, the sparsity of the global matrix is preserved. Note that, if $\hat{\mathscr{L}}=\mathscr{L}$, $\hat{\mathscr{L}}^{g}=0$ and $\tau_{e}$ is chosen properly the abstract form of the Galerkin Least-Square (GLS) [4] stabilized formulation is retrieved.

With the purpose of choosing the operators $\hat{\mathscr{L}}$ and $\hat{\mathscr{L}}^{g}$ and the functions $\tau_{e}, \tau_{e}^{g}$, we start by introducing, for each element $\Omega_{e}$, the space $E_{\mathrm{GPR}}\left(\Omega_{e}\right)$ defined as

$E_{\mathrm{GPR}}\left(\Omega_{e}\right)=\left\{\varphi: \Omega_{e} \rightarrow \mathbb{R} ; \varphi=\sum_{i=1}^{n_{\mathrm{pel}}} \sum_{j=1}^{n_{\mathrm{pel}}} C_{i j} \mathscr{L}^{*}\left(\eta_{i}^{e}\right) \mathscr{L}\left(\eta_{j}^{e}\right)\right\}$,

where $\mathscr{L}^{*}$ is an operator, $n_{\text {pel }}$ denotes the number of nodes per element, $\eta_{i}^{e}$ stands for the standard finite element polynomials restricted to the element $\Omega_{e}$, and $C_{i j}$ are real constants. Indeed, the following results are also valid when $\mathscr{L}^{*}\left(\eta_{i}^{e}\right)$ are replaced by any local polynomial. This fact can be explored for proposing different finite element formulations. To simplify the notation in what follows we use $\eta_{i}$ in place of $\eta_{i}^{e}$ whenever this does not cause any misunderstanding. To provide usefulness for the theory, the following condition is assumed for $E_{\mathrm{GPR}}\left(\Omega_{e}\right)$.

Condition 1: The space $E_{\mathrm{GPR}}\left(\Omega_{e}\right)$ contains non-trivial elements of $L^{2}\left(\Omega_{e}\right)$, or equivalently, there exist $\left(i^{\prime}, j^{\prime}\right) \in$ 
$\left\{1, \ldots, n_{\text {pel }}\right\} \times\left\{1, \ldots, n_{\text {pel }}\right\}$ such that $\mathscr{L}^{*}\left(\eta_{i^{\prime}}\right) \mathscr{L}\left(\eta_{j^{\prime}}\right) \neq 0$ in $L^{2}\left(\Omega_{e}\right)$.

The dimension of $E_{\mathrm{GPR}}\left(\Omega_{e}\right)$ (hereafter referred to as $D$ ), which is directly related to method's capabilities on improving the quality of the corresponding finite element approximation, depends on the specific problem and on the adopted finite element space.

As will be done further on, to specify $\hat{\mathscr{L}}, \hat{\mathscr{L}}^{g}$ and the functions $\tau_{e}, \tau_{e}^{g}$ it is necessary to know a base for $E_{\mathrm{GPR}}\left(\Omega_{e}\right)$. This base can be constructed as follows:

Consider $E_{v}$ as an arbitrary real linear space equipped with an inner product $(\cdot, \cdot)_{E_{v}}$ and its induced norm $\|\cdot\|_{E_{v}}$. To construct a basis for $E_{\mathrm{GPR}}\left(\Omega_{e}\right)$ the following result from linear algebra is used.

Basic result: Let $E_{v}^{0}$ be a subspace of $E_{v}$ and also let $\vartheta_{1}, \ldots, \vartheta_{J}, J$ linearly independent vectors of $E_{v}^{0}$. For any $\Psi \in E_{v}$, the real constants $\Theta_{1}(\Psi), \ldots, \Theta_{J}(\Psi)$ are computed as the solution of the following linear system

$\sum_{j=1}^{J}\left(\vartheta_{i}, \vartheta_{j}\right)_{E_{v}} \Theta_{j}(\Psi)=\left(\Psi, \vartheta_{i}\right)_{E_{v}} ; \quad i=1, \ldots, J$.

Moreover, let $\Psi^{J}$ be defined as

$\Psi^{J}=\sum_{j=1}^{J} \Theta_{j}(\Psi) \vartheta_{j}$

Then $\Psi$ and $\vartheta_{1}, \ldots, \vartheta_{J}$ form a set of linearly dependent vectors in $E_{v}$ if and only if $\left\|\Psi-\Psi^{J}\right\|_{E_{v}}=0$.

Proof. (a) If $\Psi$ and $\vartheta_{1}, \ldots, \vartheta_{J}$ are linearly dependent in $E_{v} \Rightarrow$ there exist $\theta_{1}, \ldots, \theta_{J}$ such that

$\Psi=\sum_{j=1}^{J} \theta_{j} \vartheta_{j}$

and noting that $\Psi^{J}$ given by (10) is the projection of $\Psi$ in $E_{v}$ we have

$0 \leqslant\left\|\Psi-\Psi^{J}\right\|_{E_{v}} \leqslant\left\|\Psi-\sum_{j=1}^{J} \theta_{j} \vartheta_{j}\right\|_{E_{v}}=0$.

(b) $\left\|\Psi-\Psi^{J}\right\|_{E_{v}}=0 \Rightarrow \Psi=\sum_{j=1}^{J} \Theta_{j}(\Psi) \vartheta_{j} \Rightarrow \Psi, \vartheta_{1}, \ldots, \vartheta_{J}$ are linearly dependent in $E_{v}$.

We use the above result to obtain a basis $\left\{\psi_{l}\right\}$ for $E_{\mathrm{GPR}}\left(\Omega_{e}\right)$ defining $E_{v}=L^{2}\left(\Omega_{e}\right)$ and $E_{v}^{0}=E_{\mathrm{GPR}}\left(\Omega_{e}\right)$. When it is implemented within a computer code, inherently $\varepsilon_{\text {prec }}>0$ such that $\|\varphi-\eta\|_{E_{v}} \leqslant \varepsilon_{\text {prec }}$ is utilized. Thus, the condition $\varphi-\eta=0$ should be understood as an equality within an error interval given by $\varepsilon_{\text {prec }}$. Usually, $\left(\varepsilon_{\text {prec }}\right)^{2}$ is chosen as the machine precision, for example, when using standard PC's, $\left(\varepsilon_{\text {prec }}\right)^{2}=10^{-14}$ if double precision is used. An computational algorithm for obtaining a basis for $E_{\mathrm{GPR}}\left(\Omega_{e}\right)$ is detailed in Appendix A.

At that point, it is worth to observe that GPR method can be built within a general framework that includes all GLS stabilizations. Aiming at this, the space $E_{\mathrm{GPR}}\left(\Omega_{e}\right)$ is enlarged to ensure that it contains the function $\Psi^{1}=1$ in $\Omega_{e}$. The enlarged space is referred to as $E_{\mathrm{enl}}\left(\Omega_{e}\right)$ and it is constructed as proceeded, emphasizing how to obtain a basis for the new space.

Introducing the function $\Phi^{1}=\sum_{l=1}^{D} \Theta_{l}(\Psi) \psi_{l}$, with each $\Theta_{l}(\Psi)$ obtained through solving the projection schemes (9) and (10)

$\sum_{j=1}^{D}\left(\psi_{i}, \psi_{j}\right)_{L^{2}\left(\Omega_{e}\right)} \Theta_{j}(\Psi)=\left(\Psi, \psi_{i}\right)_{L^{2}\left(\Omega_{e}\right)} ; \quad i=1, \ldots, D$

with $\Psi=1$, and the real number $\varepsilon_{1}=\left\|\Psi^{1}-\Phi^{1}\right\|_{L^{2}\left(\Omega_{e}\right)}$, the space $E_{\text {enl }}\left(\Omega_{e}\right)$ is defined as follows:

$$
\left\{\begin{array}{l}
E_{\mathrm{enl}}\left(\Omega_{e}\right)=E_{\mathrm{GPR}}\left(\Omega_{e}\right), \quad \text { if } \varepsilon_{1} \leqslant \varepsilon_{\mathrm{prec}}, \\
E_{\mathrm{enl}}\left(\Omega_{e}\right)=\left\{\eta \in L^{2}\left(\Omega_{e}\right) ; \eta=\sum_{l=0}^{D} B_{l} \psi_{l}, B_{l} \in \mathbb{R}\right\}, \text { otherwise, }
\end{array}\right.
$$

where

$\psi_{0}=1$ in $\Omega_{e}$.

Defining

$$
\left\{\begin{array}{l}
D_{\text {enl }}=D, \quad \text { if } \varepsilon_{1} \leqslant \varepsilon_{\text {prec }} \\
D_{\text {enl }}=D+1, \quad \text { otherwise }
\end{array}\right.
$$

it follows that $D_{\mathrm{enl}}$ is the dimension of $E_{\mathrm{enl}}\left(\Omega_{e}\right)$ and $\psi_{l}^{\text {enl }}\left(l=1, \ldots, D_{\text {enl }}\right)$ defined by

$\left\{\begin{array}{l}\psi_{l}^{\mathrm{enl}}=\psi_{l}, \quad l=1,2, \ldots, D, \quad \text { if } D_{\mathrm{enl}}=D \\ \psi_{1}^{\mathrm{enl}}=1, \quad \text { and } \quad \psi_{l}^{\text {enl }}=\psi_{(l-1)}, \quad l=2,3, \ldots, D_{\mathrm{enl}}\end{array}\right.$

if $D_{\text {enl }}=D+1$

provides a basis for $E_{\mathrm{enl}}\left(\Omega_{e}\right)$. It is worth to observe that the above functions are defined on the element level. Therefore, any function $\phi_{l}$ in $E_{\text {enl }}\left(\Omega_{e}\right)$ can be represented as

$\phi_{l}=\sum_{i=1}^{D_{\text {enl }}} \widehat{C}_{l i}^{e} \psi_{i}^{\text {enl }}$.

The general formulation of GPR method, introduced in (5), relies on choosing

$$
\begin{aligned}
& \mathscr{L}^{*}\left(u_{e}^{h}\right)=\mathscr{L}\left(u_{e}^{h}\right), \\
& \widehat{\mathscr{L}}^{g}\left(v_{e}^{h}\right)=\frac{\mathscr{L}\left(v_{e}^{h}\right)}{k^{4}}, \quad \widehat{\mathscr{L}}\left(v_{e}^{h}\right)=\frac{\mathscr{L}\left(v_{e}^{h}\right)}{k^{2}}, \quad \frac{\tau_{e}^{g}}{2}=\tau_{e}=\sum_{l=1}^{D_{\mathrm{enl}}} \tau_{l}^{e} \psi_{l, e}^{\mathrm{enl}}
\end{aligned}
$$

with $\tau_{l}^{e}$ for $l=1,2, \ldots, D_{\text {enl }}$, and $e=1,2, \ldots, n_{e}$ being constants to be determined. Indeed, the method is named after this specific form of the $\widehat{\mathscr{L}}$ and $\widehat{\mathscr{L}}^{g}$ once the appended terms are now recognized as a projection of the residual plus residual gradient in the $E_{\text {enl }}\left(\Omega_{e}\right)$ space. Note that by choosing $\widehat{\mathscr{L}}\left(v^{h}\right)$ and $\widehat{\mathscr{L}}^{g}\left(v^{h}\right)$ as $(20)$ the term corresponding to the projected residual plus residual gradient in (5) is a symmetric term and if the differential operator $\mathscr{L}$ is symmetric, then the GPR formulation (5) retains the symmetry of the problem. It should be highlighted that the GPR method presents great potential to make use of the advantages of the variational multiscale framework [17]. In this 
sense, the unknown $u$ can be decomposed as a sum of the unknowns in two different scales. The small scale, for example, can be explored in the context of bubbles which vanish on element boundaries and its degrees-of-freedom are eliminated by the well-known technique of static condensation.

Remark. After plugging the above choice for $\widehat{\mathscr{L}}\left(v^{h}\right)$ and $\widehat{\mathscr{L}}^{g}\left(v^{h}\right)$ into (5), the element matrix associate with GPR method is still not completely defined before fixing the values of set $\tau_{l}^{e}$. This can be carried out adopting stability and/or convergence criteria relying either on the physics of the problem (improving local balance) or on the underlying mathematics (best approximation, optimal or nearly optimal convergence, for example). For each specific choice of a criterium a new finite element method is obtained. This will be illustrated below, in detail, for Helmholtz equation.

Remark. When dealing with non-Dirichlet boundary conditions, non-homogeneous equations, transient and nonlinear problems it is of great value having a variational formulation as the one established in (5). Finite element analysis using non-structured meshes can also benefit from this general strategy for constructing stabilized finite element methods.

\section{Applying the GPR method to Helmholtz problem}

The GPR method presented in the previous section is now applied to the Helmholtz equation. Essentially, the free parameters of the method are set in order to improve the performance of the proposed method when compared to the standard Galerkin formulation.

Let $\tau_{1}^{e}, \ldots, \tau_{D_{\mathrm{ent}}}^{e}$ real constants corresponding to the function $\tau_{e}$ associated with the element $e$. The proposed finite element method consists in finding $u^{h} \in S^{h}$ that satisfies $\forall v^{h} \in V^{h}$ the variational equation,

$A_{\mathrm{GPR}}\left(u^{h}, v^{h}\right)=F_{\mathrm{GPR}}\left(v^{h}\right) \quad \forall v^{h} \in V^{h}$

with

$$
\begin{aligned}
A_{\mathrm{GPR}}\left(u^{h}, v^{h}\right)= & A\left(u^{h}, v^{h}\right)+\sum_{e=1}^{n_{e}} \sum_{l=1}^{D_{\mathrm{enl}}} \tau_{l}^{e}\left[\left(\mathscr{L}\left(u_{e}^{h}\right), \frac{\mathscr{L}\left(v_{e}^{h}\right) \psi_{l, e}^{\mathrm{enl}}}{k^{2}}\right)_{\Omega_{e}}\right. \\
& \left.+2 \int_{\Omega_{e}} \frac{\left(\nabla \mathscr{L}\left(u_{e}^{h}\right)\right) \cdot\left(\nabla \mathscr{L}\left(v_{e}^{h}\right)\right) \psi_{l, e}^{\mathrm{enl}}}{k^{4}} \mathrm{~d} \Omega\right], \\
F_{\mathrm{GPR}}^{h}\left(v^{h}\right)= & F\left(v^{h}\right)+\sum_{e=1}^{n_{e}} \sum_{l=1}^{D_{\mathrm{enl}}} \tau_{l}^{e}\left[\left(f_{e} \frac{\mathscr{L}\left(v_{e}^{h}\right) \psi_{l, e}^{\mathrm{enl}}}{k^{2}}\right)_{\Omega_{e}}\right. \\
& \left.+2 \int_{\Omega_{e}} \frac{\left(\nabla f_{e}\right) \cdot\left(\nabla \mathscr{L}\left(v_{e}^{h}\right)\right) \psi_{l, e}^{\mathrm{enl}}}{k^{4}} \mathrm{~d} \Omega\right],
\end{aligned}
$$

where $f_{e}$ denotes the restriction of $f$ to $\Omega_{e}$. Note that this finite element formulation is consistent, in the sense that the exact solution of (3) is also the solution of (21).
Let $u_{e}^{h}$ be the restriction of $u^{h}$ to $\Omega_{e}$ given by

$u_{e}^{h}=\sum_{m=1}^{n_{\mathrm{pel}}} \hat{u}_{e}^{h}(m) \eta_{m}$,

where $\hat{u}_{e}^{h}(m)$ denotes the value of $u_{e}^{h}$ in local node $m$ of $\Omega_{e}$ element. Therefore, we have

$$
\begin{aligned}
& \left(\mathscr{L}\left(u_{e}^{h}\right), \frac{\mathscr{L}\left(\eta_{i}\right) \psi_{l}^{\mathrm{enl}}}{k^{2}}\right)_{\Omega_{e}}+2 \int_{\Omega_{e}} \frac{\nabla \mathscr{L}\left(u_{e}^{h}\right) \cdot \nabla \mathscr{L}\left(\eta_{i}\right) \psi_{l, e}^{\mathrm{enl}}}{k^{4}} \mathrm{~d} \Omega \\
& =\sum_{m=1}^{n_{\mathrm{pel}}} \hat{u}_{e}^{h}(m)\left[\left(\mathscr{L}\left(\eta_{m}\right), \frac{\mathscr{L}\left(\eta_{i}\right) \psi_{l}^{\mathrm{enl}}}{k^{2}}\right)_{\Omega_{e}}\right. \\
& \left.\quad+2 \int_{\Omega_{e}} \frac{\nabla \mathscr{L}\left(\eta_{m}\right) \cdot \nabla \mathscr{L}\left(\eta_{i}\right) \psi_{l, e}^{\mathrm{enl}}}{k^{4}} \mathrm{~d} \Omega\right] \\
& i=1, \ldots, n_{\mathrm{pel}}, \quad l=1, \ldots, D_{\mathrm{enl}} .
\end{aligned}
$$

Consider $M^{l}\left(l=1, \ldots, D_{\text {enl }}\right)$ as being a set of $n_{\text {pel }} \times n_{\text {pel }}$ matrices defined as

$$
\begin{aligned}
M_{i j}^{l}= & \left(\mathscr{L}\left(\eta_{j}\right), \frac{\mathscr{L}\left(\eta_{i}\right) \psi_{l}^{\mathrm{enl}}}{k^{2}}\right)_{\Omega_{e}} \\
& +2 \int_{\Omega_{e}} \frac{\nabla \mathscr{L}\left(\eta_{j}\right) \cdot \nabla \mathscr{L}\left(\eta_{i}\right) \psi_{l, e}^{\mathrm{enl}}}{k^{4}} \mathrm{~d} \Omega \\
i= & 1, \ldots, n_{\mathrm{pel}}, \quad j=1, \ldots, n_{\mathrm{pel}} .
\end{aligned}
$$

Therefore,

$$
\begin{aligned}
& \left(\mathscr{L}\left(u_{e}^{h}\right), \frac{\mathscr{L}\left(\eta_{i}\right) \psi_{l}^{\mathrm{enl}}}{k^{2}}\right)_{\Omega_{e}}+2 \int_{\Omega_{e}} \frac{\nabla \mathscr{L}\left(u_{e}^{h}\right) \cdot \nabla \mathscr{L}\left(\eta_{i}\right) \psi_{l, e}^{\mathrm{enl}}}{k^{4}} \mathrm{~d} \Omega \\
& =\sum_{m=1}^{n_{\mathrm{pel}}} M_{i m}^{l} \hat{u}_{e}^{h}(m)
\end{aligned}
$$

and $\left[A_{\mathrm{GPR}}^{e}\right]_{i m}$ denoting entries of the element matrix detailed through

$\left[A_{\mathrm{GPR}}^{e}\right]_{i m}=A^{e}\left(\eta_{m}, \eta_{i}\right)+\sum_{l=1}^{D_{\mathrm{enl}}} \tau_{l}^{e} M_{i m}^{l}$,

whereas for the Helmholtz equation

$$
\begin{aligned}
A^{e}\left(\eta_{m}, \eta_{i}\right)= & \int_{\Omega_{e}}\left(\nabla \eta_{m} \cdot \nabla \eta_{i}-k^{2} \eta_{m} \eta_{i}\right) \mathrm{d} \Omega \\
& +\int_{\Gamma_{r} \cap \Gamma_{e}} \alpha \eta_{m} \eta_{i} \mathrm{~d} \Gamma .
\end{aligned}
$$

Remark. Numerical results indicate that the residual gradient projection term in (21) is decisive to assure uniform convergence for Helmholtz equation.

We can notice that the element matrix is formed by the usual part of Galerkin plus a projected residual with the corespondent projected residual gradient of the differential equation at element level. A possible criterion to determine the free parameters $\tau_{1}^{e}, \ldots, \tau_{D_{\text {enl }}}^{e}$, corresponding to the pro- 
jected residual with the corespondent projected residual gradient, consists of fitting the element matrix of GPR method to given matrix determined through some stability and/or convergence criteria. This matrix will be denominated GPR-generating matrix and denoted by $M^{\text {gen }}$.

For Helmholtz equation with uniform mesh and bilinear quadrilateral elements we have the basis $\psi_{1}, \ldots, \psi_{D}$ for $E_{\mathrm{GPR}}\left(\Omega_{e}\right)$

$$
\begin{aligned}
& \left(-\nabla \cdot \nabla \eta_{1}-k^{2} \eta_{1}\right)\left(-\nabla \cdot \nabla \eta_{1}-k^{2} \eta_{1}\right)=\psi_{1}=+k^{4} \eta_{1} \eta_{1}, \\
& \left(-\nabla \cdot \nabla \eta_{1}-k^{2} \eta_{1}\right)\left(-\nabla \cdot \nabla \eta_{2}-k^{2} \eta_{2}\right)=\psi_{2}=+k^{4} \eta_{1} \eta_{2}, \\
& \left(-\nabla \cdot \nabla \eta_{1}-k^{2} \eta_{1}\right)\left(-\nabla \cdot \nabla \eta_{3}-k^{2} \eta_{3}\right)=\psi_{3}=+k^{4} \eta_{1} \eta_{3}, \\
& \left(-\nabla \cdot \nabla \eta_{1}-k^{2} \eta_{1}\right)\left(-\nabla \cdot \nabla \eta_{4}-k^{2} \eta_{4}\right)=\psi_{4}=+k^{4} \eta_{1} \eta_{4}, \\
& \left(-\nabla \cdot \nabla \eta_{2}-k^{2} \eta_{2}\right)\left(-\nabla \cdot \nabla \eta_{2}-k^{2} \eta_{2}\right)=\psi_{5}=+k^{4} \eta_{2} \eta_{2}, \\
& \left(-\nabla \cdot \nabla \eta_{2}-k^{2} \eta_{2}\right)\left(-\nabla \cdot \nabla \eta_{3}-k^{2} \eta_{3}\right)=\psi_{6}=+k^{4} \eta_{2} \eta_{3}, \\
& \left(-\nabla \cdot \nabla \eta_{3}-k^{2} \eta_{3}\right)\left(-\nabla \cdot \nabla \eta_{3}-k^{2} \eta_{3}\right)=\psi_{7}=+k^{4} \eta_{3} \eta_{3}, \\
& \left(-\nabla \cdot \nabla \eta_{3}-k^{2} \eta_{3}\right)\left(-\nabla \cdot \nabla \eta_{4}-k^{2} \eta_{4}\right)=\psi_{8}=+k^{4} \eta_{3} \eta_{4}, \\
& \left(-\nabla \cdot \nabla \eta_{4}-k^{2} \eta_{4}\right)\left(-\nabla \cdot \nabla \eta_{4}-k^{2} \eta_{4}\right)=\psi_{9}=+k^{4} \eta_{4} \eta_{4} .
\end{aligned}
$$

$D_{\mathrm{enl}}=D=9$ and $\tau_{l}^{e}=\tau_{l} \forall \Omega_{e}$. Observe that $\eta_{2} \eta_{4}=\eta_{1} \eta_{3}$.

For the homogeneous Helmholtz equation, uniform meshes and Dirichlet boundary condition the element matrix $M^{\mathrm{QS}}$ that minimizes the phase error is associated to the stencil given in [3]. In this case, it is interesting to choose the GPR-generating matrix as

$M^{\text {gen }}=\Upsilon_{3} M^{\mathrm{QS}}$,

where $\Upsilon_{3}$ is a parameter that should be determined and the matrix $M^{\mathrm{QS}}$ is

$$
M^{\mathrm{QS}}=\left[\begin{array}{cccc}
\tau_{1}^{*} & \tau_{2}^{*} & \tau_{3}^{*} & \tau_{4}^{*} \\
\tau_{2}^{*} & \tau_{5}^{*} & \tau_{6}^{*} & \tau_{3}^{*} \\
\tau_{3}^{*} & \tau_{6}^{*} & \tau_{7}^{*} & \tau_{8}^{*} \\
\tau_{4}^{*} & \tau_{3}^{*} & \tau_{8}^{*} & \tau_{9}^{*}
\end{array}\right],
$$

being that the $\tau_{i}^{*}$ can be determined though the standard dispersion analysis following the steps:

(1) From typical dispersion analysis, a plane wave solution $\mathrm{e}^{(\mathrm{i} \tilde{k}(x \cos \theta+y \sin \theta))}(\mathrm{i}=\sqrt{-1}, 0 \leqslant \theta \leqslant \pi)$ propagating in the $\theta$ with wave number $\tilde{k}$ is imposed to the interior stencil of GPR, yielding

$$
\begin{aligned}
\bar{\tau}_{0} & +\bar{\tau}_{1} \cos (\tilde{k} h \sin \theta)+\bar{\tau}_{2} \cos (\tilde{k} h \cos \theta) \\
& +\bar{\tau}_{3} \cos (\tilde{k} h \sin \theta) \cos (\tilde{k} h \sin \theta)=0,
\end{aligned}
$$

with

$$
\begin{aligned}
& \bar{\tau}_{0}=\tau_{1}^{*}+\tau_{5}^{*}+\tau_{7}^{*}+\tau_{9}^{*}, \\
& \bar{\tau}_{1}=2\left(\tau_{4}^{*}+\tau_{6}^{*}\right), \\
& \bar{\tau}_{2}=2\left(\tau_{2}^{*}+\tau_{8}^{*}\right), \\
& \bar{\tau}_{3}=4 \tau_{3}^{*} .
\end{aligned}
$$

Notice that the parameters $\bar{\tau}_{0}, \bar{\tau}_{1}, \bar{\tau}_{2}$ and $\bar{\tau}_{3}$ depend on $k$ but not on $\tilde{k}$. The stencil defined in (31) is a linear algebraic equation with four unknowns $\bar{\tau}_{0}, \bar{\tau}_{1}, \bar{\tau}_{2}$ and $\bar{\tau}_{3}$. Choosing two different directions $\theta_{1}$ and $\theta_{2}$ for the plane wave the interior stencil generates two linearly independent equations. Thus, two unknowns are still undetermined within the dispersion analysis.

(2) Due to the mesh symmetric, the following restrictions for the free parameters can be imposed

$$
\begin{aligned}
& \tau_{1}^{*}=\tau_{5}^{*}=\tau_{7}^{*}=\tau_{9}^{*}=\frac{1}{4} \Rightarrow \bar{\tau}_{0}=1, \\
& \tau_{4}^{*}=\tau_{6}^{*}=\frac{1}{2} \bar{\tau}_{1} \text { and } \tau_{2}^{*}=\tau_{8}^{*}=\frac{1}{2} \bar{\tau}_{2}=\frac{1}{2} \bar{\tau}_{1} \Rightarrow \bar{\tau}_{1}=\bar{\tau}_{2} .
\end{aligned}
$$

It should be emphasized that for uniform meshes only two free parameters are necessary to retrieve the optimal stencil obtained in [3]. For non-uniform meshes these restrictions cannot be imposed, since the mesh is not symmetrical. With the imposed restrictions, the interior stencil leads to

$$
\begin{aligned}
1+ & \bar{\tau}_{1}(\cos (\tilde{k} h \sin \theta)+\cos (\tilde{k} h \cos \theta)) \\
& +\bar{\tau}_{3} \cos (\tilde{k} h \sin \theta) \cos (\tilde{k} h \sin \theta)=0 .
\end{aligned}
$$

(3) Minimizing the phase error of the approximate solution, following the work [3], yields

$$
\begin{aligned}
& \bar{\tau}_{1}=\frac{\left(r_{1}-r_{2}\right)}{\left(r_{2} w_{1}-r_{1} w_{2}\right)}, \\
& \bar{\tau}_{3}=\frac{\left(w_{2}-w_{1}\right)}{\left(r_{2} w_{1}-r_{1} w_{2}\right)}
\end{aligned}
$$

with

$$
\begin{aligned}
& r_{1}=\cos \left(k h \cos \frac{\pi}{16}\right) \cos \left(k h \sin \frac{\pi}{16}\right), \\
& r_{2}=\cos \left(k h \cos \frac{3 \pi}{16}\right) \cos \left(k h \sin \frac{3 \pi}{16}\right), \\
& w_{1}=\cos \left(k h \cos \frac{\pi}{16}\right)+\cos \left(k h \sin \frac{\pi}{16}\right), \\
& w_{2}=\cos \left(k h \cos \frac{3 \pi}{16}\right)+\cos \left(k h \sin \frac{3 \pi}{16}\right) .
\end{aligned}
$$

Therefore, the GPR-generating matrix $M^{\text {gen }}$ corresponding to the matrix given in [3] is

$$
M^{\text {gen }}=\Upsilon_{3}\left[\begin{array}{cccc}
\frac{1}{4} & \frac{\bar{\tau}_{1}}{2} & \frac{\bar{\tau}_{3}}{4} & \frac{\bar{\tau}_{1}}{2} \\
\frac{\bar{\tau}_{1}}{2} & \frac{1}{4} & \frac{\bar{\tau}_{1}}{2} & \frac{\bar{\tau}_{3}}{4} \\
\frac{\bar{\tau}_{3}}{4} & \frac{\bar{\tau}_{1}}{2} & \frac{1}{4} & \frac{\bar{\tau}_{1}}{2} \\
\bar{\tau}_{1} & \frac{\bar{\tau}_{3}}{2} & \frac{\bar{\tau}_{1}}{4} & \frac{1}{4}
\end{array}\right] \text {. }
$$

The stencil equation (34) establishes an implicit relation between $k$ and $k$. Only in one-dimension this relationship can be established explicitly. On the other hand, solving numerically (34) one can find an approximate expression through expanding $\tilde{k}$ in Taylor's series for each $k h$ and $\theta$ fixed. A $k h$ that verifies the thumb rule was chosen. The coefficients of Taylor expansion about point $k h=\frac{2 \pi}{10}$ are present in Table 1. The propagation direction $\theta$ was chosen such that the largest phase error for GPR method $\left(\theta=\frac{\pi}{4}\right)$ would occur. The coefficients of Taylor expansion for the asymptotically optimal interior stencil $(k h \rightarrow 0)$ are presented in [3]. The asymptotical interior stencil of GPR method coincides with 
Table 1

Coefficients of Taylor's series for $\tilde{k} h$

\begin{tabular}{|c|c|c|c|}
\hline \multirow[t]{2}{*}{$n$} & \multicolumn{3}{|c|}{$f(x)=\sum_{n=0}^{4} \frac{f^{(n)}\left(x_{o}, \theta\right)}{n !}\left(x-x_{o}\right)^{n}+\mathrm{O}\left(x^{5}\right)$, where $x=k h, x_{o}=\frac{2 \pi}{10}$ and $\theta=\frac{\pi}{4}$} \\
\hline & $f^{(n)}\left(x_{o}\right)$ for Galerkin FEM & $f^{(n)}\left(x_{o}\right)$ for GLS Method & $f^{(n)}\left(x_{o}\right)$ for GPR Method \\
\hline 0 & 0.623263068938366 & 0.630860911418061 & 0.628318583528965 \\
\hline 1 & 0.976211478991646 & 1.011999929416810 & 1.000000597871875 \\
\hline 2 & $-7.298797423795446 \times 10^{-2}$ & $3.704483546481942 \times 10^{-2}$ & $5.8479509493624245 \times 10^{-6}$ \\
\hline 3 & $-9.928933682143666 \times 10^{-2}$ & $5.110641691485787 \times 10^{-2}$ & $4.834592828857333 \times 10^{-5}$ \\
\hline 4 & 0.363094317513384 & -0.199439802149905 & $3.009745987903417 \times 10^{-4}$ \\
\hline
\end{tabular}

the asymptotically optimal interior stencil presented in [3]. Using the expansion

$$
\begin{aligned}
\frac{\tilde{k}-k}{k}= & {\left[f^{(1)}\left(x_{o}, \theta\right)-1\right]+\frac{\left[f\left(x_{o}, \theta\right)-\frac{2 \pi}{10} f^{(1)}\left(x_{o}, \theta\right)\right]}{k h} } \\
& +\frac{f^{(2)}\left(x_{o}, \theta\right)}{2} \frac{\left(k h-\frac{2 \pi}{10}\right)^{2}}{k h}+\mathrm{O}\left(\left(\frac{k h-\frac{2 \pi}{10}}{\sqrt[3]{k h}}\right)^{3}\right),
\end{aligned}
$$

and considering Table 1 we have

- For GPR method:

$$
\begin{aligned}
\frac{\tilde{k}-k}{k} \approx & 5.9787187 \times 10^{-7}-\frac{3.2284297 \times 10^{-7}}{k h}+2.9239754 \\
& \times 10^{-6} \frac{\left(k h-\frac{2 \pi}{10}\right)^{2}}{k h} .
\end{aligned}
$$

- For GLS method:

$$
\begin{aligned}
\frac{\tilde{k}-k}{k} \approx & 1.1999929 \times 10^{-2}-\frac{4.9973973 \times 10^{-3}}{k h}+1.8522417 \\
& \times 10^{-2} \frac{\left(k h-\frac{2 \pi}{10}\right)^{2}}{k h} .
\end{aligned}
$$

- For Galerkin method:

$$
\begin{aligned}
\frac{\tilde{k}-k}{k} \approx & -2.3788521 \times 10^{-2}+\frac{9.8913067 \times 10^{-3}}{k h} \\
& -3.6493987 \times 10^{-2} \frac{\left(k h-\frac{2 \pi}{10}\right)^{2}}{k h} .
\end{aligned}
$$

To determine the nine free parameters $\tau_{l}^{e}$ in (26) by fitting the element matrix of GPR method to $M^{\text {gen }}$ we considered the $J$ functional defined as

$J=\sum_{m=1}^{n_{\mathrm{pel}}} \sum_{i=1}^{n_{\mathrm{pel}}}\left[A^{e}\left(\eta_{m}, \eta_{i}\right)+\left(\sum_{l=1}^{D_{\mathrm{enl}}} \tau_{l} M_{i m}^{l}\right)-M_{i m}^{\mathrm{gen}}\right]^{2}$.

Due to the mesh symmetric, the following restrictions for the free parameters can be imposed again

$$
\begin{aligned}
& \tau_{1}=\tau_{5}=\tau_{7}=\tau_{9}=0, \\
& \tau_{2}=\tau_{4}=\tau_{6}=\tau_{8}=\Upsilon_{1}, \\
& \tau_{3}=\Upsilon_{2}
\end{aligned}
$$

Therefore, the $J$ functional can be written as

$$
\begin{aligned}
J= & \sum_{m=1}^{n_{\mathrm{pel}}} \sum_{i=1}^{n_{\mathrm{pel}}}\left[A^{e}\left(\eta_{m}, \eta_{i}\right)+\Upsilon_{1}\left(M_{i m}^{2}+M_{i m}^{4}+M_{i m}^{6}+M_{i m}^{8}\right)\right. \\
& \left.+\Upsilon_{2} M_{i m}^{3}-\Upsilon_{3} M_{i m}^{\mathrm{QS}}\right]^{2}
\end{aligned}
$$

Finally, for each $\Omega_{e}$ the parameters $\Upsilon_{1}, \Upsilon_{2}$ and $\Upsilon_{3}$ are determined solving the following system of algebraic equations:

$\frac{\partial J}{\partial \Upsilon_{m}}=0, \quad m=1,2,3$.

It should be observed that for the parameters $\Upsilon_{1}, \Upsilon_{2}, \Upsilon_{3}$ and $\tau_{l}$ determined by (42) and (44), the element matrix of GPR method coincides with the GPR generating matrix.

Remark. A GPR method is derived for each particular choice of the set of free parameters $\tau_{l}^{e}$. Usually, these parameters are determined through a dispersion analysis of the finite element approximation restricted to uniform meshes. In this sense, when a dispersion analysis is applied to the GPR method the stencil of the Quasi Stabilized Finite Element Method [3] can be retrieved for a certain choice of the stabilization parameters. It is well known that the element matrix associated to this stencil minimizes the phase error in relation to uniform meshes. Also, it is well known that a FEM with two free parameters can retrieve this stencil. Since there is no notice about an optimal stencil for nonuniform meshes, choosing free parameters of stabilized finite element formulation applied to Helmholtz equation is an open question for unstructured meshes, in general. And the optimal values of the stabilization parameters, determined for uniform mesh, are surely not optimal for non-uniform meshes. If an element matrix associated to optimal stencil for non-uniform meshes were known (GPR-generating matrix), the free parameters could be chosen to retrieve this stencil. Since non-uniform meshes are not symmetrical, to retrieve this kind of stencil one should expect a FEM formulation with more than two free parameters. In this case, a FEM with a greater number of free parameters has a greater capability to retrieve this optimal stencil. We are working on the development of alternative strategies applied to nonuniform meshes and whatever is the progress it will be presented in the next paper.

\section{Numerical results}

In the present section, a number of examples are presented to illustrate the main features and potential of 
GPR method applied to Helmholtz equation. The first group of numerical tests deals with homogeneous Helmholtz equation and the second group of numerical tests deals with inhomogeneous Helmholtz equation. At that point, before presenting the numerical results, it is convenient to recall that Helmholtz problem is not well posed for a number of exciting frequencies corresponding to the resonant modes. Also, since the GPR method is applied here to interior Helmholtz problem and examples with Dirichlet boundary conditions are presented, care must be taken to avoid numerical resonance. Particularly, at high wave numbers the resonances tend to cluster and a FEM method with a slight degree of dispersion (phase error of the method) can lead to great errors due to the vicinity of numerical resonance. Some numerical evaluations of resonance for Helmholtz problems appear in [19]. Here for $k L=100$ the corresponding values of the numerical resonance were avoided in the numerical experiments presented below. In all examples a unity square domain, bilinear shape functions, $3 \times 3$ Gaussian integration, uniform mesh $(160 \times 60)$ and the same wave number $(k=100)$ are adopted.

The first group of numerical test deals with plane waves propagating in 2-D domains. As the propagation direction is not known a priori, the free parameters are the ones computed in the previous section. Three 2-D examples are presented to show the importance of having a finite element formulation capable to minimize the phase error for homogeneous Helmholtz equation. These examples are illustrated as the accuracy and stability of some FEM with large phase errors (such as Galerkin and GLS methods) deteriorate and are compared with stabilized formulations that are able to minimize the phase error (such as QS, DGB, RBFEM and GPR methods). It should be highlighte that for uniform meshes and homogeneous equation the solutions of QS, DGB and GPR methods coincide.

The first example of this group of numerical tests has Dirichlet boundary conditions such that the exact solution

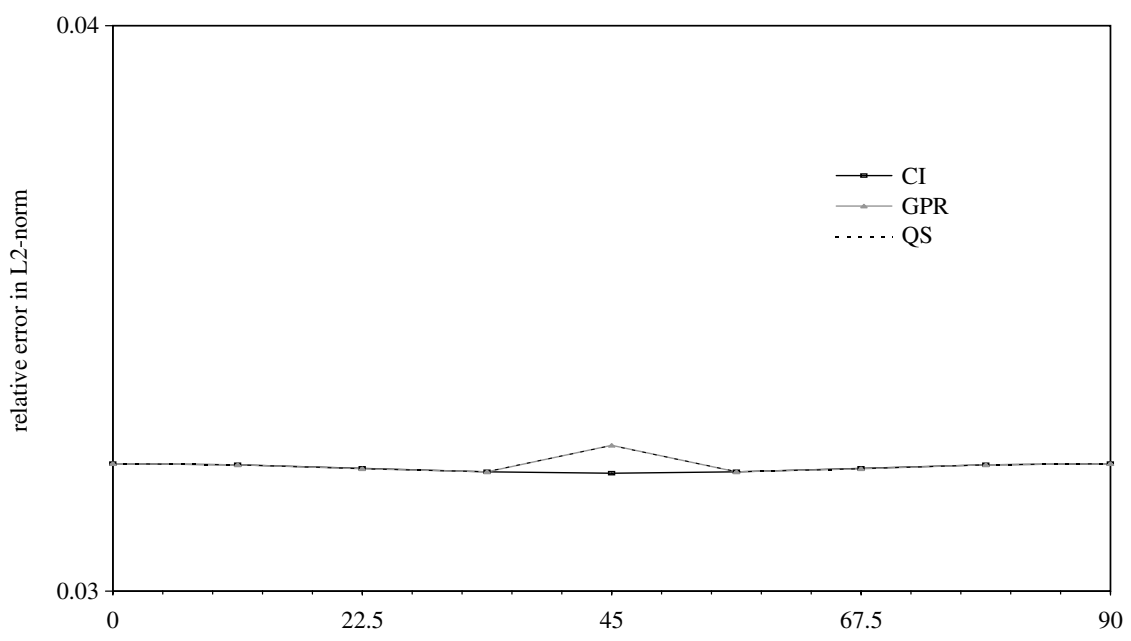

Fig. 1. Relative error of the CI, GPR and QS solutions in the $L^{2}$-norm as a function of $\theta$-direction.

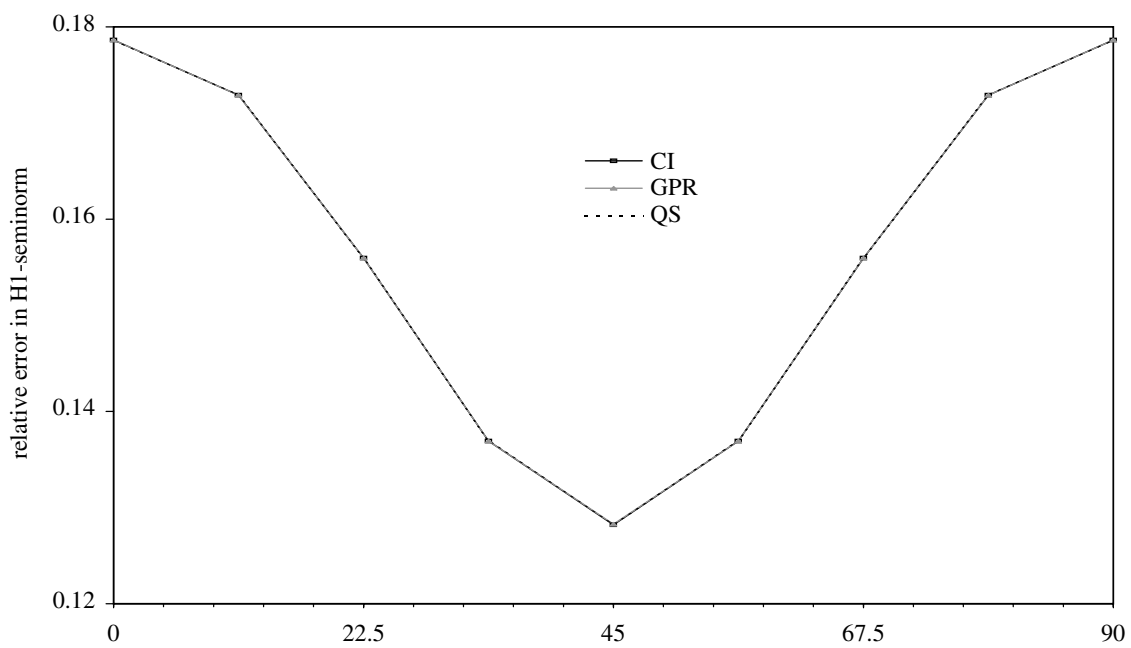

Fig. 2. Relative error of the CI, GPR and QS solutions in the $H^{1}$-seminorm as a function of $\theta$-direction. 
is a plane wave propagating in $\theta$-direction, $u(x, y)=\cos (k(x \cos \theta+y \sin \theta))$. In all examples of this group of numerical tests the stabilization parameter of GLS method is determined by eliminating the phase error in the direction $\theta=\frac{\pi}{8}$, as proposed in [5].

Figs. 1 and 2 present a comparison between the relative errors in $L^{2}$-norm and $H^{1}$-seminorm of the GPR, continuous interpolant $(\mathrm{CI})$ and QS solutions. In this case, the solutions of the QS and GPR methods coincide. Fig. 3 shows the nodal interpolant, GPR and GLS solutions in sections $x=0.5$ along the $y$ direction for $\theta=\frac{\pi}{4}$ for this example.

The next example is similar to the previous one, but now the exact solution is given by a superposition of $n$ monoenergetic plane waves propagating in $n$ different $\theta$-directions, $u(x, y)=\sum_{i=1}^{n} \cos \left(k\left(x \cos \theta_{i}+y \sin \theta_{i}\right)\right)$. Firstly, three plane waves propagating in the directions $\theta_{1}=0, \theta_{2}=\frac{\pi}{8}$ and $\theta_{3}=\frac{\pi}{4}$ are considered. The relative errors in $L^{2}$-norm, $H^{1}$ seminorm and $H^{1}$-norm are presented in Table 2. Fig. 4 shows the nodal interpolant, GPR and GLS solutions in sections $x=0.5$ along the $y$ direction. Fig. 5 shows the same FEM solutions in section $y=0.5$ along the $x$ direction. Again, the results show the good performance of the GPR formulation and the reduction of the phase error over all wave vector orientations $\theta$ by this formulation.

Secondly, six plane waves propagating in the directions $\theta_{1}=0, \theta_{2}=\frac{\pi}{20}, \theta_{3}=\frac{\pi}{10}, \theta_{4}=\frac{3 \pi}{20}, \theta_{5}=\frac{\pi}{5}$ and $\theta_{6}=\frac{\pi}{4}$ are considered. Figs. 6 and 7 show the nodal interpolant, GPR and GLS solutions in sections $x=0.5$ and $y=0.5$, respectively. Again, relative errors in $L^{2}$-norm, $H^{1}$-seminorm and $H^{1}$-norm are presented in Table 2 . Very similar conclusions to the previous example can be drawn. We should observe that in these two examples the directions of plane waves propagations are always different from $\theta_{1}=\frac{\pi}{16}$ and $\theta_{2}=\frac{3 \pi}{16}$, which are the directions for asymptotically optimal interior stencil.

The second group of numerical tests has source term (inhomogeneous Helmholtz equation) and Dirichlet
Table 2

Relative errors of FEMs for three and six plane waves

\begin{tabular}{lllll}
\hline \multicolumn{1}{c}{ CI } & GPR & GLS & Galerkin \\
\hline $\begin{array}{l}\text { Relative errors of three finite element methods } \\
\text { Three plane waves }\end{array}$ & & \\
$L^{2}$-norm & $3.22 \mathrm{E}-02$ & $3.23 \mathrm{E}-02$ & $5.40 \mathrm{E}-01$ & $1.71 \mathrm{E}+00$ \\
$H^{1}$-seminorm & $1.56 \mathrm{E}-01$ & $1.56 \mathrm{E}-01$ & $5.59 \mathrm{E}-01$ & $1.72 \mathrm{E}+00$ \\
$H^{1}$-norm & $1.56 \mathrm{E}-01$ & $1.56 \mathrm{E}-01$ & $5.59 \mathrm{E}-01$ & $1.72 \mathrm{E}+00$ \\
Six plane waves & & & & \\
$L^{2}$-norm & $3.22 \mathrm{E}-02$ & $3.23 \mathrm{E}-02$ & $5.45 \mathrm{E}-01$ & $3.24 \mathrm{E}+00$ \\
$H^{1}$-seminorm & $1.56 \mathrm{E}-01$ & $1.56 \mathrm{E}-01$ & $5.69 \mathrm{E}-01$ & $3.24 \mathrm{E}+00$ \\
$H^{1}$-norm & $1.56 \mathrm{E}-01$ & $1.56 \mathrm{E}-01$ & $5.69 \mathrm{E}-01$ & $3.24 \mathrm{E}+00$ \\
\hline
\end{tabular}

boundary conditions such that the exact solution is a plane wave propagating in $\theta$-direction plus a polynomial function, $u(x, y)=p(x, y)+\sin (k(x \cos \theta+y \sin \theta))$. In the first example, $p(x, y)=x+y$ (case 1), the second example $p(x, y)=x^{2}+y^{2}$ (case 2) and the third example $p(x, y)=$ $(1+x+y)^{3} \quad$ (case 3). That is, $f(x, y)=-k^{2}(x+y)$, $f(x, y)=-4-k^{2}\left(x^{2}+y^{2}\right)$ and $f(x, y)=-12(1+x+y)-$ $k^{2}(1+x+y)^{3}$, respectively. In Figs. 8 and 9 , the errors of the GPR method in L2-norm and H1-seminorm relative to the continuous bilinear interpolant are presented, respectively. The GPR approximation is very close to the continuous interpolant for any $\theta$-direction of plane wave. Notice that for case 1, the errors of the GPR method just have the part corresponding to the error of the plane wave, since the bilinear shape functions approximate the linear polynomial function exactly. Fig. 10 shows the GPR solutions in sections $x=0.5$ along the $y$ direction obtained with $\theta=\frac{\pi}{4}$ for cases 1 and 2 of the source term. These results clearly show that the GPR solution is very close to the exact solution for this $\theta$-direction of plane wave which corresponds to the direction of the largest phase lag for GPR approximation.

A convergence study is carried out for non-homogeneous Helmholtz equation and we observe a uniform convergence, independently of the value of the wave number $k$.

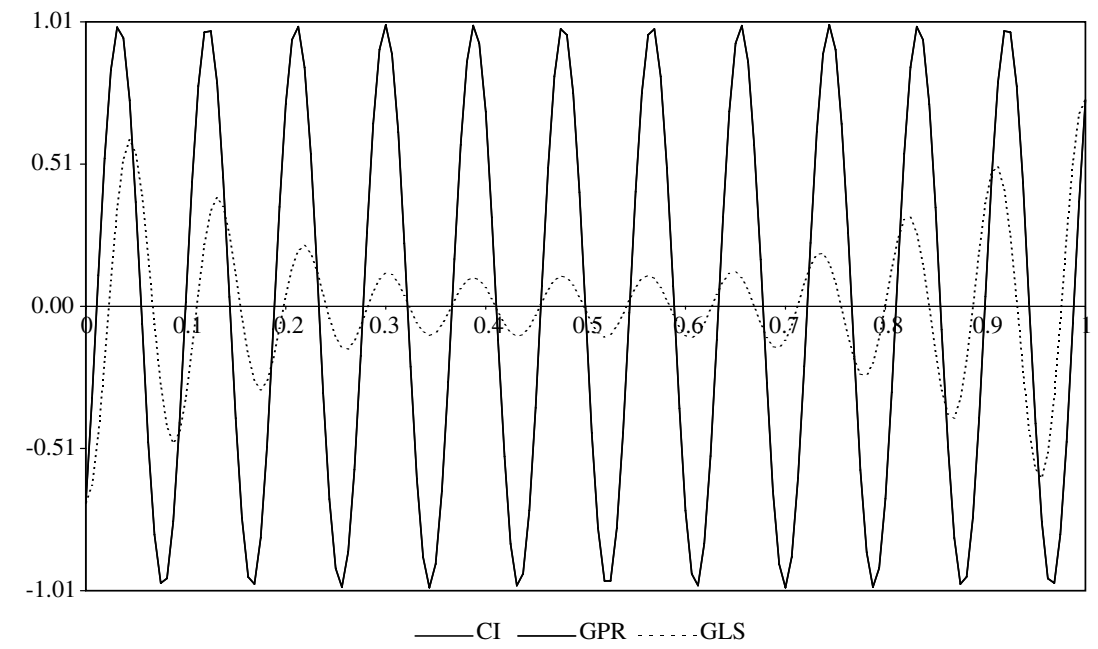

Fig. 3. Solution of homogeneous problem in two-dimensions at sections $x=0.5$ for $\theta=\frac{\pi}{4}$. 


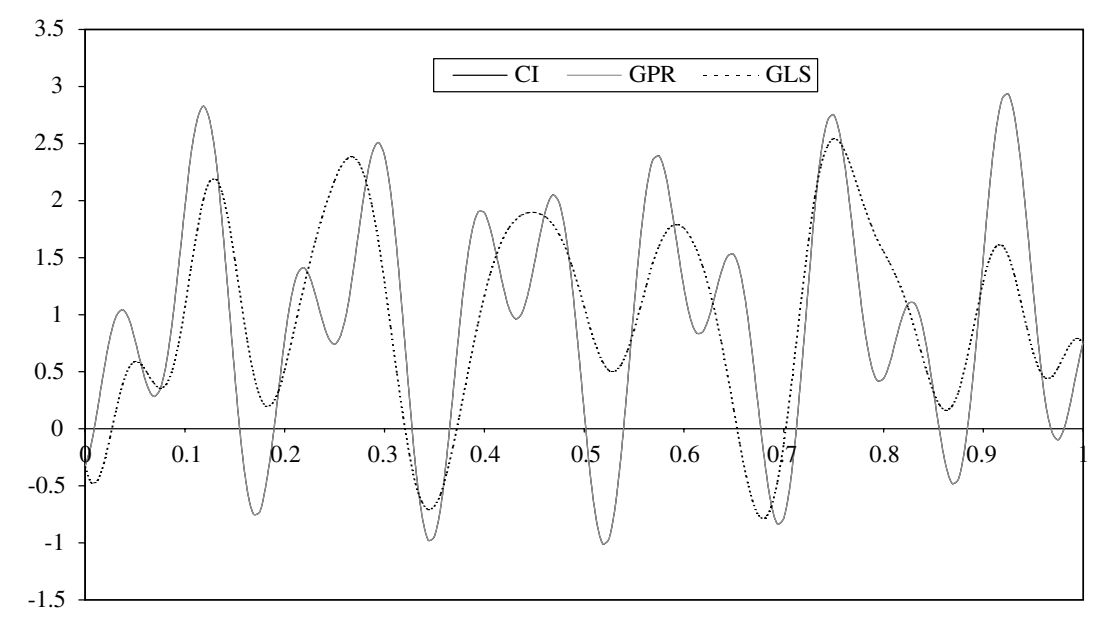

Fig. 4. GPR and GLS solutions of homogeneous problem in two-dimensions at sections $x=0.5$, three plane waves.

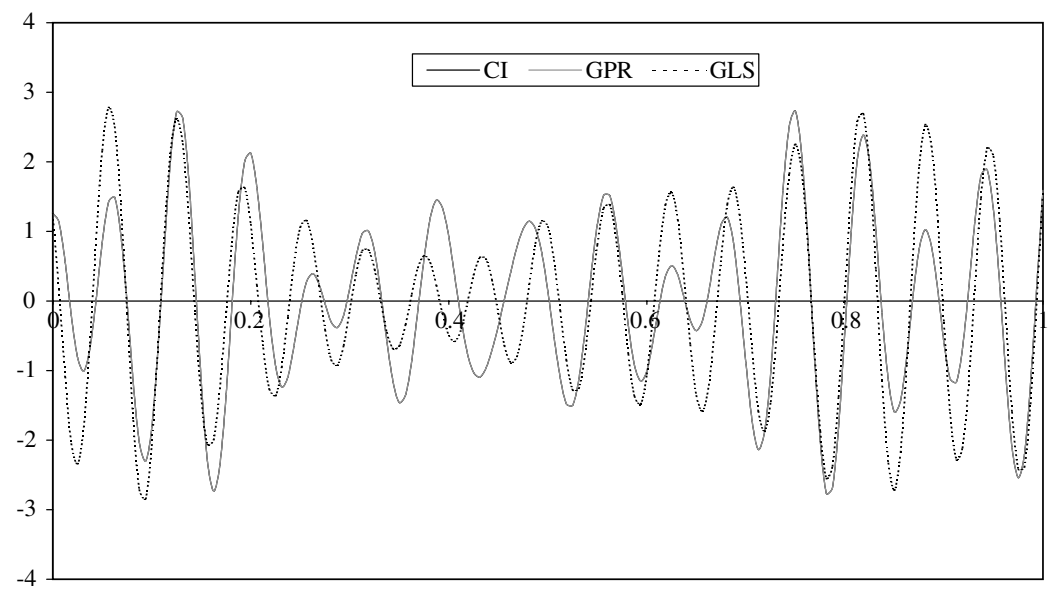

Fig. 5. GPR and GLS solutions of homogeneous problem in two-dimensions at sections $y=0.5$, three plane waves.

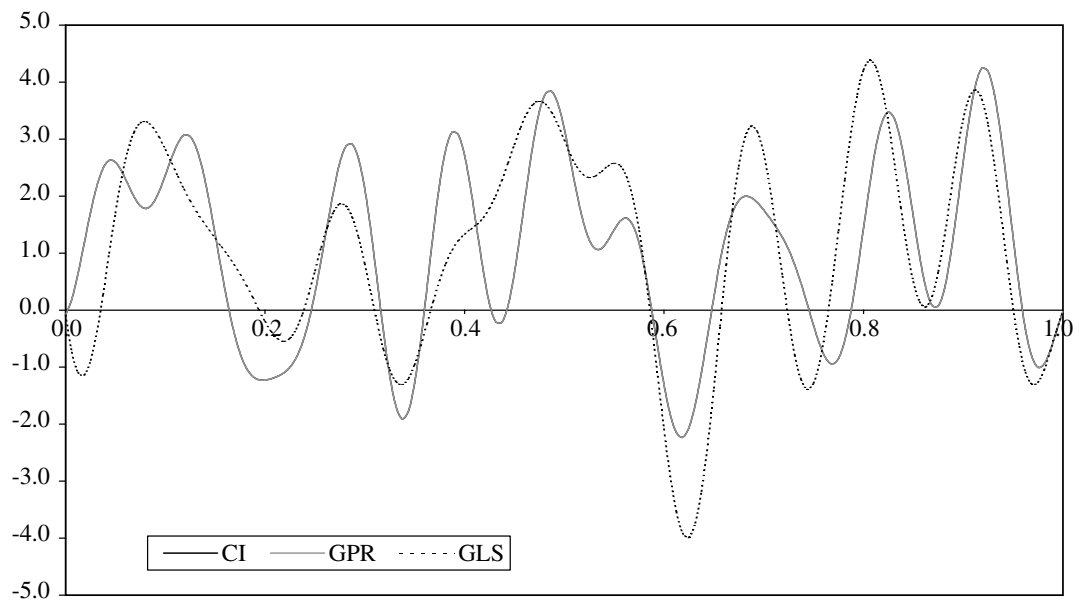

Fig. 6. GPR and GLS solutions of homogeneous problem in two-dimensions at sections $x=0.5$, six plane waves.

Fig. 11 presents, for cases 2 and 3 with $k=10$, the errors of the GPR solutions in the $L^{2}$-norm and $H^{1}$-seminorm as a function of $h$ relative to continuous interpolant. A uniform refinement is employed starting with a $10 \times 10$ mesh until a 


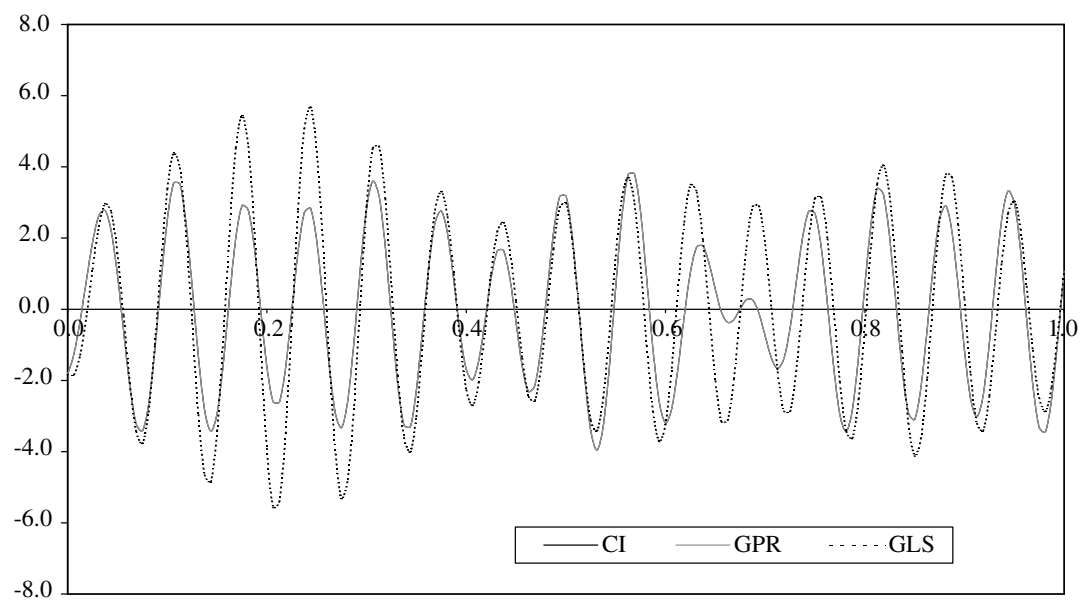

Fig. 7. GPR and GLS solutions of homogeneous problem in two-dimensions at sections $y=0.5$, six plane waves.

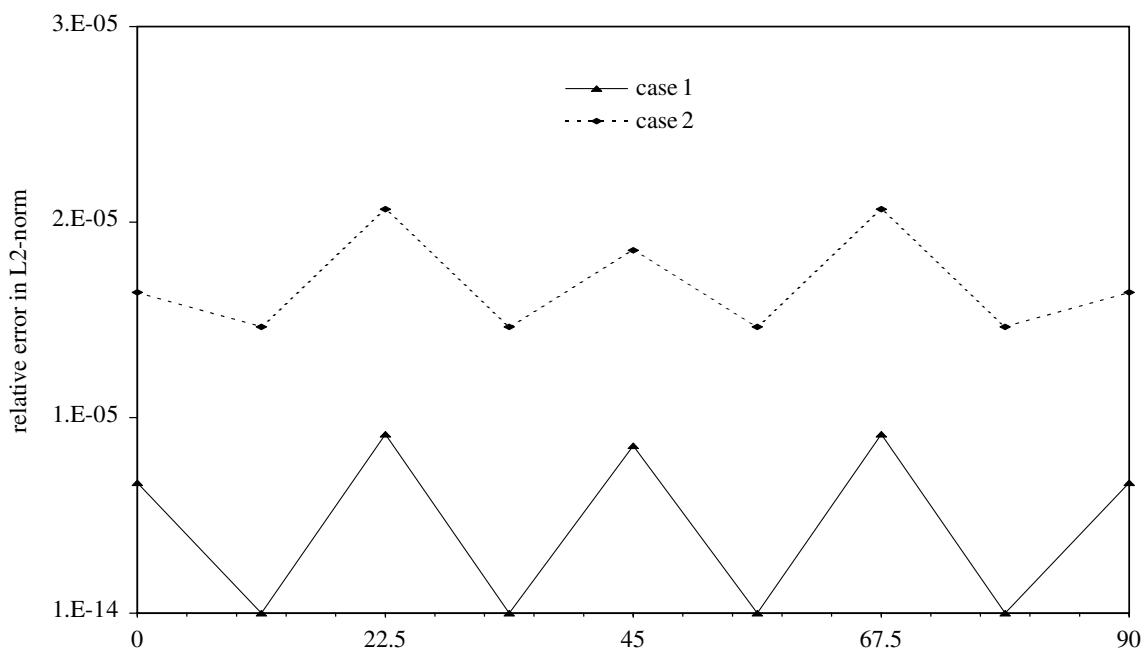

Fig. 8. Non-homogeneous Helmholtz equation. Error of the GPR solutions in the $L^{2}$-norm as a function of $\theta$-direction relative to continuous interpolant: case $1 f(x, y)=-k^{2}(x+y)$ and case $2 f(x, y)=-4-k^{2}\left(x^{2}+y^{2}\right)$.

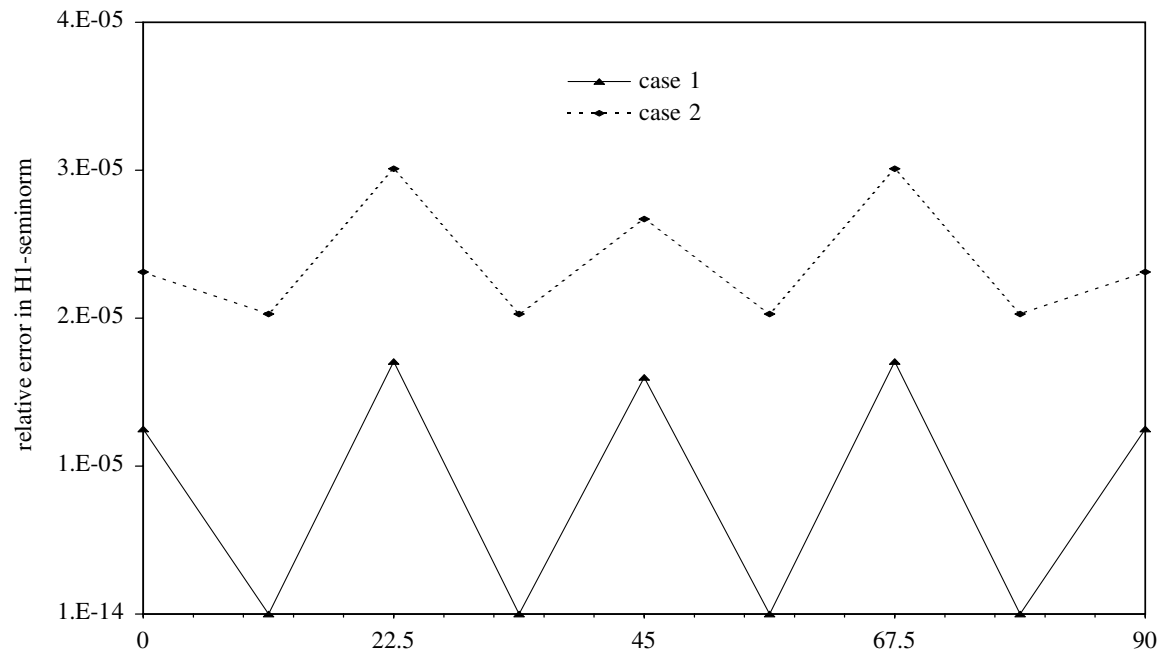

Fig. 9. Non-homogeneous Helmholtz equation. Error of the GPR solutions in the $H^{1}$-seminorm as a function of $\theta$-direction relative to continuous interpolant: case $1 f(x, y)=-k^{2}(x+y)$ and case $2 f(x, y)=-4-k^{2}\left(x^{2}+y^{2}\right)$. 


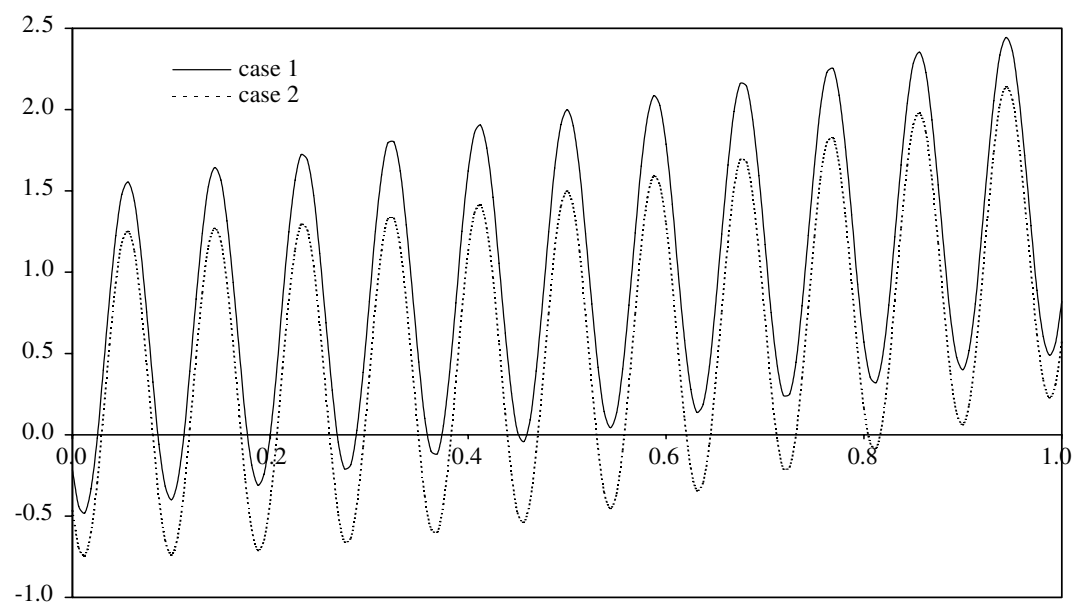

Fig. 10. Non-homogeneous Helmholtz equation. GPR solutions in two-dimensions at sections $x=0.5$ for $\theta=\frac{\pi}{4}$ : case $1 f(x, y)=-k^{2}(x+y)$ and case 2 $f(x, y)=-4-k^{2}\left(x^{2}+y^{2}\right)$.

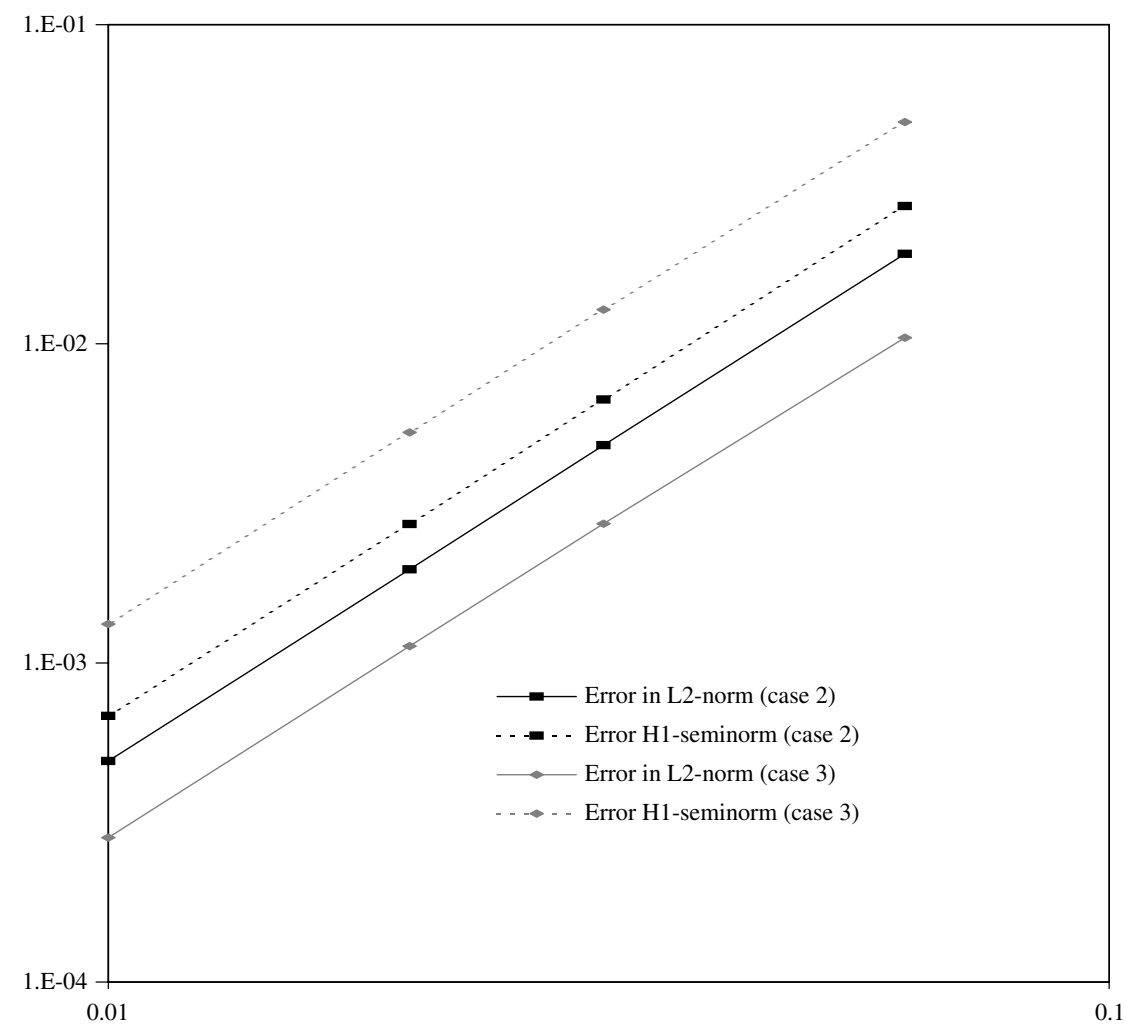

Fig. 11. Convergence study for non-homogeneous Helmholtz equation. Error of the GPR solutions in the $L^{2}$-norm and $H^{1}$-seminorm as a function of $h$ relative to continuous interpolant for cases 2 and 3 with $k=10$.

$100 \times 100$ mesh. The results show good rates of convergence for the GPR approximation.

\section{Final considerations}

A consistent stabilized $C^{0}$ finite element formulation for Helmholtz problem valid for any dimension and for any order of polynomials is presented.
For each operator and local approximation space this method introduces a set of free parameters via a weighted residual added to the Galerkin formulation. The free parameters can be determined by appropriate criteria allowing to find the nearly optimal finite element approximation to the Helmholtz problem in the infinite dimensional space $S$. For uniform meshes, explicit values of the free parameters minimizing the phase error are obtained via a dispersion analysis. 
For bilinear shape functions the GPR method applied to Helmholtz equation needs $3 \times 3$ Gaussian integration, since the local base of $E_{\mathrm{GPR}}\left(\Omega_{e}\right)$ is quadratic polynomials. Even so, the GPR method only has volume terms. The extra computational effort of GPR method is minor compared to more complex stabilized finite element formulations existing in the literature, and even some simple ones such as RBFEM and DGB methods.

The numerical simulations presented here emphasize the importance of having a FEM that minimizes the phase error consistently. Indeed, different paths have been pursued and recently, by exploring a multiscale prospective [17], a stable method with good results was achieved in [12] for Helmholtz problem.

The good performance of the proposed formulation obtained for Helmholtz equation stimulates us to apply the GPR method to other problems in future works.

\section{Acknowledgements}

The authors wish to thank the Brazilian research funding agencies $\mathrm{CNPq}$ and FAPERJ for their support to this work.

\section{Appendix A. Algorithm for constructing a basis for $E_{\mathrm{GPR}}(e)$}

This appendix contains a computational algorithm to constructing a basis for $E_{\mathrm{GPR}}\left(\Omega_{e}\right)$, which is described below.

(a) Procedure to determine the function $\operatorname{Lig}(\circ, \circ)$. compute $N_{t}=n_{\text {pel }}\left(n_{\text {epel }}+1\right) / 2$

define $l=0$

FOR $i=1, N_{t}$

FOR $j=i, N_{t}$ compute $l=l+1$

$\operatorname{Lig}(l, 1)=i$

$\operatorname{Lig}(l, 2)=j$

END FOR $j$

END FOR $i$

(b) Procedure to define the functions $\psi_{1}, \ldots, \psi_{N_{t}}$.

FOR $l=1, N_{t}$

compute $\psi_{l}=\mathscr{L}^{*}\left(\eta_{\operatorname{Lig}(l, 1)}\right) \mathscr{L}\left(\eta_{\operatorname{Lig}(l, 2)}\right)$

END FOR $l$

(c) Procedure to obtain the basis $\psi_{1}^{b}, \ldots, \psi_{D}^{b}$

define $D=1$

define $\varepsilon=10^{-7}$

define $\psi_{1}^{b}=\psi_{1}$

FOR $l=1, N_{t}$

FOR $i=1, D$

FOR $j=1, D$

compute $A_{i j}=\left(\psi_{i}^{b}, \psi_{j}^{b}\right)_{L^{2}\left(\Omega_{e}\right)}$

compute $A_{j i}=A_{i j}$

END FOR $j$

compute $b_{i}=\left(\psi_{l}, \psi_{i}^{b}\right)_{L^{2}\left(\Omega_{e}\right)}$

END FOR $i$

\author{
solve the system $\sum_{j=1}^{D} A_{i j} \theta_{j}=b_{i}(i=1, \ldots, D)$ \\ compute $\left\|\psi_{l}-\sum_{j=1}^{D} \theta_{j} \psi_{j}^{b}\right\|_{L^{2}\left(\Omega_{e}\right)}$ \\ IF $\left\|\psi_{l}-\sum_{j=1}^{D} \theta_{j} \psi_{j}^{b}\right\|>\varepsilon$, then \\ compute $D=D+1$ \\ define $\psi_{D}^{b}=\psi_{l}$ \\ END IF \\ END FOR $l$
}

\section{References}

[1] F. Ihlenburg, I. Babuška, Finite element solution of the Helmholtz equation with high wave number Part I: the h-version of the FEM, Comput. Math. Appl. 30 (9) (1995) 9-37.

[2] I. Harari, T.J.R. Hughes, Finite element method for the Helmholtz equation in an exterior domain: model problems, Comp. Meth. Appl. Mech. Eng. 87 (1991) 59-96.

[3] I. Babuška, F. Ihlenburg, E.T. Paik, S.A. Sauter, A generalized finite element method for solving the Helmholtz equation in two dimensions with minimal pollution, Comput. Methods Appl. Mech. Engrg. 128 (1995) 325-359.

[4] I. Harari, T.J.R. Hughes, Galerkin/least squares finite element methods for the reduced wave equation with non-reflecting boundary conditions in unbounded domains, Comp. Methods Appl. Mech. Engrg. 98 (1992) 411-454.

[5] L.L. Thompson, P.M. Pinsky, A Galerkin least squares finite element method for the two-dimensional Helmholtz equation, Int. J. Numer. Methods Engrg. 38 (3) (1995) 371-397.

[6] L.L. Thompson, P.M. Pinsky, in: E. Stein, R. de Borst, T.J.R. Hughes (Eds.), Acoustics: Encyclopedia of Computational Mechanics, John Wiley \& Sons, Ltd, (C2004.

[7] A.A. Oberai, P.M. Pinsky, A residual-based finite element method for the Helmholtz equation, Int. J. Numer. Methods Engrg. 49 (2000) 399-419.

[8] A.F.D. Loula, G.B. Alvarez, E.G. Dutra do Carmo, F.A. Rochinha, A discontinuous finite element method at element level for Helmholtz equation, Comput. Methods Appl. Mech. Engrg. 196 (2007) 867-878.

[9] F.A. Rochinha, G.B. Alvarez, E.G. Dutra do Carmo, A.F.D. Loula, A locally discontinuous enriched finite element formulation for acoustics, Commun. Numer. Methods Engrg. 23 (2007) 623-637.

[10] P.E. Barbone, I. Harari, Nearly H1-optimal finite element methods, Comput. Methods Appl. Mech. Engrg. 190 (43) (2001) 56795690 .

[11] A. Nesliturk, I. Harari, The nearly-optimal Petrov-Galerkin method for convection-diffusion problems, Comput. Methods Appl. Mech. Engrg. 192 (22) (2003) 2501-2519.

[12] I. Harari, K. Gosteev, Bubble-based stabilization for the Helmholtz equation, Int. J. Numer. Methods Engrg. 70 (2007) 1241-1260.

[13] L.P. Franca, C. Farhat, A.P. Macedo, M. Lesoinne, Residual-free bubbles for the Helmholtz equation, Int. J. Numer. Methods Engrg. 40 (1997) 4003-4009.

[14] C. Farhat, I. Harari, U. Hetmaniuk, A discontinuous Galerkin method with Lagrange multipliers for the solution of Helmholtz problems in the mid-frequency regime, Comput. Methods Appl. Mech. Engrg. 192 (2003) 1389-1419.

[15] G.B. Alvarez, A.F.D. Loula, E.G. Dutra do Carmo, F.A. Rochinha, A discontinuous finite element formulation for the Helmholtz equation, Comput. Methods Appl. Mech. Engrg. 195 (2006) 4018 4035.

[16] E.G. Dutra do Carmo, G.B. Alvarez, F.A. Rochinha, A.F.D. Loula, A new finite element formulation: the Galerkin Projected Residual method (GPR). The GPR method applied to diffusive-reactive problems, Comput. Methods Appl. Mech. Engrg. (submitted for publication). 
[17] T.J.R. Hughes, Multiscale phenomena: Green's functions, the Dirichlet-to-Neumann formulation, subgrid scale models, bubbles and the origins of stabilized methods, Comput. Methods Appl. Mech. Engrg. 127 (1/4) (1995) 387-401.

[18] E.G. Dutra do Carmo, G.B. Alvarez, A.F.D. Loula, F.A. Rochinha, The Galerkin plus multiplies projection of residual method (GMPR) applied to Helmholtz equation, in: Proceedings of the XXVII Iberian Latin-American Congress on Computational Methods in Engineering - CILAMCE 2006, Belém do Pará, Brazil.
[19] F. Ihlenburg, Finite element analysis of acoustic scattering, Applied Mathematical Sciences, vol. 132, Springer-Verlag, New York, 1998.

[20] Theofanis Strouboulis, Ivo Babuška, Realino Hidajat, The generalized finite element method for Helmholtz equation: theory, computation, and open problems, Comput. Methods Appl. Mech. Engrg. 195 (2006) 4711-4731.

[21] L.P. Franca, E.G.D. Do Carmo, The Galerkin gradient least squares method, Comput. Methods Appl. Mech. Engrg. 74 (1989) 41-54. 